\title{
BRCA1 : des percées et des énigmes?
}

Trois articles récents présentent des études fonctionnelles du gène $B R C A 1$ dont les mutations germinales sont associées à une prédisposition au cancer du sein et de l'ovaire [1].

L'inactivation du gène Brcal chez la souris produit des animaux hétérozygotes $\left[\mathrm{Brcal}^{+/-}\right]$normaux, fertiles, sans prédisposition à développer des tumeurs. En revanche, chez les animaux nullizygotes [ $\mathrm{Brcal}^{-/-}$], l'embryogenèse est interrompue au jour 6,57,5 . Cet effet létal avant la gastrulation semble lié à l'absence de prolifération et donc de développement des différents feuillets embryonnaires. Il interdit de reproduire chez ces animaux le processus de cancérogenèse mammaire observé chez les femmes porteuses d'une prédisposition héréditaire liée à des mutations de brca1. La technologie de mutagenèse conditionnelle produit aujourd'hui un modèle assez proche de la pathologie humaine reproduisant le processus à deux coups du modèle classique de Knudson [2]. Les souris sont porteuses d'un premier allèle de brcal constitutionnellement inactivé et d'un second allèle entouré de sites loxP. Le croisement de ces souris avec des souris transgéniques pour le gène Cre permet l'excision de l'exon 11 et l'apparition de la mutation. L'expression de Cre étant sous le contrôle d'un promoteur spécifique du tissu épithélial mammaire, soit le promoteur MMTV soit le promoteur Wap (whey acidic protein), l'inactivation du second allèle brcal ne se produira que dans ce tissu. L'inactivation de brcal retentit sur le développement de la glande mammaire, de la grossesse, de la lactation et de l'involution avec pour conséquence une morphogenèse très incomplète de la glande, caractérisée par une inhibition du développement canalaire et une apoptose anor- malement élevée. Lorsqu'ils atteignent un âge de 10 à 13 mois, environ $30 \%$ des animaux développent divers types de tumeurs dont l'histopathologie est très semblable à celle des tumeurs humaines. Ces tumeurs sont caractérisées par une aneuploïdie marquée avec des anomalies récurrentes du chromosome 11, en particulier une perte de $p 53$. L'importance de cet événement est renforcée par le résultat de l'expérience montrant une accélération (8 mois) de la genèse des tumeurs dans un contexte génétique $p 53^{\mathrm{null} / \mathrm{+}}$, ces tumeurs ayant très fréquemment perdu l'allèle p53 sauvage.

L'absence fonctionnelle de brcal chez l'animal adulte conduit donc à deux effets apparemment contradictoires: d'une part, un effet antiprolifératif sur le tissu épithélial mammaire et, d'autre part, un effet favorisant l'éclosion d'une prolifération maligne dans ce même tissu. Cette contradiction n'est qu'apparente si l'on considère que l'absence de brcal instaure un état facilitant les erreurs de réplication, ce qui se manifeste par une instabilité génétique. Cet "état d'alarme" est capable d'activer p53 et de bloquer la division cellulaire. Ce modèle peut également rendre compte de l'échec de l'activité proliférative nécessaire au développement des différents feuillets embryonnaires chez les embryons mutants ${ }^{\text {null/null. }}$. Dans un contexte génétique déficient en p53 «l'état d'alarme" n'est plus perçu et la prolifération tumorale ou embryonnaire est permise.

Dans ce modèle, le ciblage tissulaire de l'inactivation du second allèle sur le tissu épithélial mammaire ne permet évidemment pas d'explorer l'une des questions majeures que soulève la spécificité d'organe observée chez les patients porteurs d'une mutation de brcal (sein et ovaire chez la femme et, dans une moindre mesure prostate chez l'homme). Fan et al. [3] tentent d'aborder ce problème dans une série d'expériences dont l'interprétation conduit à proposer que la protéine BRCA1 inhibe la prolifération des cellules épithéliales mammaires contrôlée par les œstrogènes. L'inactivation de cette fonction expliquerait cette prolifération inappropriée. Ces expériences montrent que la stimulation, par le $17 \beta$ ostradiol, d'un promoteur ERE-TK (estrogen receptor element-thymidine kinase) couplé au gène luciférase, est inhibée en présence de la protéine BRCA1. Cet effet est obtenu lorsque le promoteur est introduit dans des cellules tumorales de tissus mammaire et prostatique, mais pas dans des cellules de tumeurs cervicales, suggérant une spécificité tissulaire compatible avec le spectre de tumeurs observé chez les individus porteurs de mutation germinale de brca1. Les effets observés sont quantitativement importants, et les auteurs s'efforcent de montrer qu'il ne s'agit pas d'une répression non spécifique. Cependant ces expériences ne sont pas totalement convaincantes: elles ont été réalisées par transfection transitoire in vitro, et surtout elles n'explorent pas si une des formes mutées de BRCA1, forme qui est associée à une prédisposition héréditaire au cancer du sein, a aussi perdu cette propriété de répression, ce qui constitue un contrôle important pour l'extrapolation du modèle.

Alors que l'absence de brcal conduit à l'apoptose des cellules épithéliales mammaires chez les souris brcal $1^{\text {null/null, }}$ Harkin $e t$ al. proposent que ce même phénomène peut résulter cette fois de la surexpression de BRCA1 dans des cellules humaines [4]. D'autres auteurs avaient précédemment montré un tel effet sans toutefois en 
détailler les mécanismes. Ici l'analyse par oligonucléotides arrays $(\mathrm{m} / \mathrm{s} 1998$, $\left.n^{\circ} 10, p .1097\right)$ des ARNm induits en réponse à l'expression de Brcal par l'intermédiaire d'un vecteur inductible par le système Tet-Off $(\mathrm{m} / \mathrm{s} 1999$, $n^{\circ} 5, p$. 755), a permis d'identifier $G A D D 45$, un gène de réponse au stress. L'induction de ce gène est apparemment indépendante de p53. Il est établi que GADD45 peut induire l'apoptose par la voie de transmission du signal impliquant JNK/SAPK, et Harkin et al. s'attachent à démontrer que l'apoptose induite par BRCA1 est bien contrôlée par cette voie $(\mathrm{m} / \mathrm{s}$
1995, $\left.n^{\circ} 11, p .1617\right)$. Ces observations renforcent les tenants du «tout facteur de transcription» mais créent des contradictions en ce qui concerne l'apoptose.

1. Feunten J. La prédisposition héréditaire au cancer du sein liée à $B R C A 1$ et $B R C A 2$ : une maladie de la réponse aux lésions génotoxiques? Med Sci 1999 ; 15: 38-44.

2. Xu X, Wagner KU, Larson D, et al. Conditional mutation of Brcal in mammary epithelial cells results in blunted ductal morphogenesis and tumour formation. Nat Genet 1999 ; 22 : 37-43.
3. Fan S, Wang J, Yuan R, et al. BRCA1 inhibition of estrogen receptor signaling in transfected cells. Science 1999 ; 284 : 1354-6.

4. Harkin DP, Bean JM, Miklos D, et al. Induction of GADD45 and JNK/SAPK-dependent apoptosis following inducible expression of BRCA1. Cell $1999 ; 97$ : 575-86.

\section{Jean Feunteun}

Laboratoire de génétique oncologique, Cnrs UMR 1599, Institut GustaveRoussy, 39, rue Camille-Desmoulins, 94805 Villejuif Cedex, France.
Benzène et cancer : quels sont les individus "à risque " ? L'exposition au benzène, professionnelle ou environnementale, peut entraîner une hématotoxicité due à la production de métabolites qui subissent des phénomènes d'auto-oxydation et d'activation par des peroxydases. Le métabolisme $\mathrm{du}$ benzène produit une hydroxyquinone (HQ) qui, en l'absence de $\mathrm{NAD}(\mathrm{P}) \mathrm{H}$ :quinone oxydoréductase (NQO1) (ou DTdiaphorase) est oxydée en quinones toxiques. A ce titre, la NQO1, qui maintient sous forme réduite ces composés, contribue à l'action des systèmes de défense anti-oxydante. Un polymorphisme génétique $(\mathrm{C} \rightarrow \mathrm{T}$; Pro609Ser $)$ est responsable d'une très faible activité de NQO1. Il est retrouvé chez environ $20 \%$ des sujets caucasiens. Or, une susceptibilité accrue des cellules souches hématopoiétiques de la moelle à la toxicité induite par le benzène est corrélée, parmi d'autres causes, à un déficit en NQO1. Une étude réalisée chez des ouvriers chinois exposés au benzène a montré l'augmentation du risque d'hématotoxicité et de leucémie chez les individus porteurs du génotype déficient $\mathrm{T} / \mathrm{T}$ [1]. Ces malades porteurs du géno- type déficient $\mathrm{T} / \mathrm{T}$ développent davantage de leucémies secondaires à des chimiothérapies que les malades avec le génotype $\mathrm{C} / \mathrm{C}$. Un mécanisme possible est maintenant proposé [2] : les auteurs ont mis en évidence dans une lignée leucémique d'origine humaine (KG-1a) l'induction de l'activité NQO1 par HQ, ainsi que l'effet cytoprotecteur de cette induction. Ils ont pu relier l'absence d'induction de NQO1 à une toxicité accrue dans des cellules de moelle de génotype $\mathrm{T} / \mathrm{T}$ exposées aux HQ. Les mécanismes moléculaires de cette induction enzymatique ne sont pas connus; les HQ et d'autres métabolites du benzène pourraient agir via l'élément de réponse antioxydante (ARE) en relation avec la production de peroxyde d'hydrogène observée dans les cellules incubées avec des HQ. Cette présence de NQO1, après induction dans les cellules cibles de la toxicité de certains xénobiotiques, apporte un support biologique expérimental aux études épidémiologiques sur les facteurs génétiques de risque de pathologies dues à l'exposition à des xénobiotiques, comme certains cancers [3] ou des maladies neurodégénératives.
[1. Rothman N, et al. Cancer Res $1997 ; 57: 2839-42$.

[2. Moran JL, et al. Proc Natl Acad Sci USA 1999; 96 : 8150-5 (et commentaire p. 7624).]

[3. Chen H, et al. Cancer Res 1999; 59: 3045-8.]

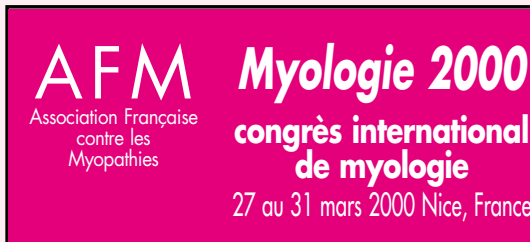

En mars 2000, l'AFM organise à Nice son premier congrès international de myologie. Son objectif : témoigner de la renaissance de la myologie en tant que discipline médicale et scientifique à part entière. Pendant 5 jours, elle réunira tous les meilleurs spécialistes internationaux de cette discipline : biologistes, physiologistes, médecins, scientifiques traiteront de tous les aspects du muscle.

Renseignements - Inscriptions

AFM - Myologie 2000

Secrétariat Permanent du Conseil Scientifique 13, place de Rungis - 75013 PARIS

Tél. : 0144162700 - Fax : 0145803736 e-mail : dduguet@mail.afm.genethon.fr 